\title{
A laboratory comparison of the interactions between 3 plastic mulch types and 38 active substances found in pesticides
}

\author{
Nicolas Beriot ${ }^{\text {Corresp., } 1,2}$, Paul Zomer ${ }^{3}$, Raul Zornoza ${ }^{2}$, Violette Geissen ${ }^{1}$ \\ ${ }^{1}$ Soil Physics and Land Management Group, Wageningen University and Research, Wageningen, Netherlands \\ Sustainable Use, Management and Reclamation of Soil and Water Research Group, Universidad Politécnica de Cartagena, Cartagena, Murcia, Spain \\ 3 Wageningen Food Safety Research, Wageningen University and Research, Wageningen, Netherlands \\ Corresponding Author: Nicolas Beriot \\ Email address: nicolas.beriot@wur.nl
}

Background. In semi-arid regions, the use of plastic mulch and pesticides in conventional agriculture is nearly ubiquitous. Although the sorption of pesticides on Low Density Polyethylene (LDPE) has been previously studied, no data are available for other plastics such as Pro-oxidant Additive Containing (PAC) plastics or "biodegradable" (Bio) plastics. The aim of this research was to measure the sorption pattern of active substances from pesticides on LDPE, PAC and Bio plastic mulches and to compare the decay of the active substances in the presence and absence of plastic debris.

Methods. For this purpose, 38 active substances from 17 insecticides, 15 fungicides and 6 herbicides commonly applied with plastic mulching in South-east Spain were incubated with a $3 \times 3 \mathrm{~cm}^{2}$ piece of plastic mulch (LDPE, PAC and Bio). The incubation was done in a solution of $10 \%$ acetonitrile and $90 \%$ distilled water at $35^{\circ} \mathrm{C}$ for 15 days in the dark. The QuEChERS (Quick Easy Cheap Effective Rugged Safe) approach was adapted to extract the pesticides.

Results. The sorption behavior depended on both the pesticide and the plastic mulch type. On average, the sorption percentage was $\sim 23 \%$ on LDPE and PAC and $\sim 50 \%$ on Bio. The decay of active substances in the presence of plastic was $\sim 30 \%$ lesser than the decay of active substances in solution alone. This study is the first attempt at assessing the behavior of a diversity of plastic mulches and pesticides to further define research needs. 
1 A laboratory comparison of the interactions between 23 plastic mulch types and 38 active substances found 3 in pesticides

4

5

6

\author{
Nicolas Beriot ${ }^{1,2}$, Paul Zomer ${ }^{3}$, Raul Zornoza ${ }^{2}$, Violette Geissen ${ }^{1}$ \\ ${ }^{1}$ Soil Physics and Land Management Group, Wageningen University and Research, \\ Wageningen, Gelderland, Netherlands \\ 2 Sustainable Use, Management and Reclamation of Soil and Water Research Group, \\ Universidad Politécnica de Cartagena, Cartagena, Murcia, Spain. \\ ${ }^{3}$ Wageningen Food Safety Research, Wageningen University and Research, Wageningen, \\ Gelderland, Netherlands \\ Corresponding author: \\ Nicolas Beriot ${ }^{1,2}$ \\ Droevendaalsesteeg 4, Wageningen, Gelderland, 6700 AE, Netherlands \\ Email address: nicolas.beriot@wur.nl
}

\title{
Abstract
}

Background. In semi-arid regions, the use of plastic mulch and pesticides in conventional agriculture is nearly ubiquitous. Although the sorption of pesticides on Low Density Polyethylene (LDPE) has been previously studied, no data are available for other plastics such as Pro-oxidant Additive Containing (PAC) plastics or "biodegradable" (Bio) plastics. The aim of this research was to measure the sorption pattern of active substances from pesticides on LDPE, PAC and Bio plastic mulches and to compare the decay of the active substances in the presence and absence of plastic debris.

Methods. For this purpose, 38 active substances from 17 insecticides, 15 fungicides and 6 herbicides commonly applied with plastic mulching in South-east Spain were incubated with a $3 \times 3 \mathrm{~cm}^{2}$ piece of plastic mulch (LDPE, PAC and Bio). The incubation was done in a solution of $10 \%$ acetonitrile and $90 \%$ distilled water at $35^{\circ} \mathrm{C}$ for 15 days in the dark. The QuEChERS (Quick Easy Cheap Effective Rugged Safe) approach was adapted to extract the pesticides. Results. The sorption behavior depended on both the pesticide and the plastic mulch type. On average, the sorption percentage was $\sim 23 \%$ on LDPE and PAC and $\sim 50 \%$ on Bio. The decay of active substances in the presence of plastic was $\sim 30 \%$ lesser than the decay of active substances in solution alone. This study is the first attempt at assessing the behavior of a diversity of plastic mulches and pesticides to further define research needs. 
52

53

54

55

56

57

58

59

60

61

62

63

64

65

66

67

68

69

70

71

72

73

74

75

76

77

78

79

80

81

82

83

84

85

86

87

88

89

\section{Introduction}

The use of plastic mulching has become a well-established technique to increase the profitability of many crops (Kasirajan and Ngouajio 2012). The European Commission estimated in 2016 that 100000 tons of plastic mulch is used per year in the European Union (European Commission 2016). Plastic mulch is generally used for one or all of the following three reasons: decreasing evaporation, decreasing weed competition or increasing soil temperature (Steinmetz et al. 2016). After crop harvest, some farmers try to remove the plastic mulch but debris is left in the soil. Other farmers simply incorporate the plastic into the soil (Kasirajan and Ngouajio 2012). Once the plastic is in the environment, the low degradation rate of plastic debris facilitates its accumulation (Rillig 2012).

The plastic mulch degradation process can be explained by looking at three main underlying factors: abiotic conditions, microbial requirements, and properties of the plastic mulch material (Hayes et al. 2012). The most common plastic used for mulching is Low Density Polyethylene (LDPE) (Kasirajan and Ngouajio 2012). LDPE is a fully saturated polymer of hydrocarbons which makes it highly resistant (Crawford et al. 2017a). Consequently, LDPE mulch needs to be removed after harvest and LDPE debris accumulates in the environment. Some plastic producers have tried to improve the degradation processes of plastic to avoid plastic mulch removal and plastic debris accumulation. Pro-oxidant Additive Containing (PAC) plastics are polymers, mainly LDPE, which contain a pro-oxidant additive that is used to enhance oxidation and photo-degradation (Selke et al. 2015). In the presence of light and under aerobic conditions, PAC plastics degrade quickly into small pieces. Small fragmented debris is more likely to be further degraded by microorganisms (Ahmed et al. 2018). PAC plastics are also known as "oxo-degradable" or "oxo-biodegradable" (Hogg 2016). However, when incorporated into the soil, the degradation process is minimized due to the absence of UV-light (Hogg 2016) and PAC debris may accumulate. Over the last few years, new mulching films that can be degraded by microorganisms in the soil have been developed (Hayes et al. 2017, Sintim and Flury 2017). They are usually sold as "biodegradable" (Bio) mulch (Oever et al. 2017). Biodegradable mulch can be made of a diversity of polymers (Kijchavengkul and Auras 2008) either biobased, synthetic or a blend of both. Biodegradation of polymeric mulch films relies on three fundamental steps: the colonization of the polymer surfaces by soil microorganisms, the enzymatic depolymerization of the polymer by extracellular hydrolases secreted by the colonizing microorganisms and the microbial utilization of the hydrolysis products that are released from the polymer (Sander 2019). Therefore, a larger contact area helps colonization and polymers containing functional groups that can be enzymatically hydrolyzed increase the degradation rate. About 3000 tons of biodegradable plastic mulch are used each year in the European Union (European Commission 2016). In order to properly manufacture plastic mulches, additives such as nucleating agents, plasticizers, performance additives, and lubricants are required (Briassoulis 2004, Hayes et al. 2012, Shen et al. 2010). It is important to note that manufacturers do not normally share the chemical structures of the raw materials or the additives that are used in plastic production in order to protect their products from being duplicated.

In arid and semi-arid areas, where water deficits are common, the use of plastic mulching for irrigated crops is widespread. It is a technically and economically feasible strategy used to prevent evaporation and reduce water consumption. This is the case in regions such as the Loess plateau in China (Jiang et al. 2016) and in the Murcia Region of South-eastern Spain (van der Meulen et al. 2006). In addition to plastic mulch, pesticides are used in conventional agriculture 
90 to control weeds, insects and fungi (Oever et al. 2017). The synergetic effect of plastic debris and

91 pesticide residues on degradation and on the terrestrial environment is not sufficiently

92 understood. Nerin et al. (1996) and Sharom and Solomon (1981) studied the sorption rates of

93 nine different active substances in pesticides on LDPE and found a sorption rate between $20 \%$

94 and $100 \%$ after 15 days at $35^{\circ} \mathrm{C}$. Adsorbed active substances are less likely to be degraded

95 (Kasirajan and Ngouajio 2012) and may be released when ingested by an organism (Teuten et al.

96 2007). Furthermore, microplastics may be carriers for pesticide residues when transported

97 through the terrestrial environment. The modification of the degradation patterns of active

98 substances might affect the soil organism community due to the toxicity of the active substances.

99 Moreover, the microbial activity plays a major role in Bio plastic degradation. Therefore,

100 adsorption of active substances could potentially decrease plastic debris degradation.

101

102

103

104

105

106

107

108

109

110

111

112

113

114

115

116

117

118

119

120

121

122

123

124

125

126

127

128

129

130

131

132

133

134

Previous studies determined the occurrence of adsorbed organic contaminants (Polycyclic aromatic hydrocarbons, organochlorinates) on different plastic polymers (Crawford et al. 2017b, Hirai et al. 2011, Mato et al. 2001) and have characterized the sorption of different organic contaminants on plastics (Lee et al. 2014, Liu et al. 2019, Mato et al. 2001, Ramos et al. 2015, Seidensticker et al. 2018, Teuten et al. 2007). Most studies were focused on the aquatic environment and coastal areas but Hüffer and al. (2019) showed that the sorption on polyethylene microplastics influenced the transport of hydrophobic organic pollutants in soils (Seidensticker et al. 2018). More data are required to assess the sorption of commonly used pesticides on LDPE and on new types of plastic.

As a preliminary investigation to address these data gaps, the sorption of 38 active substances from 17 insecticides, 15 fungicides and 6 herbicides commonly used along with plastic mulching in South-eastern Spain, were tested on three types of plastic mulch: LDPE, PAC and Bio. The objectives of this research were to measure the sorption of a mixture of 38 active substances on plastic mulch and to compare the decay of adsorbed and non-adsorbed active substances. We hypothesized that sorption rates would be different for each specific active substance and each specific plastic. For example, the Bio mulch was believed to be the most prone to sorb active substances (Boivin et al. 2005, Crawford et al. 2017a). Furthermore, we hypothesized that sorption would reduce the degradation of active substances (Nerín et al. 1996, Ramos et al. 2015).

\section{Materials \& Methods}

A laboratory single point sorption experiment was set up to test the sorption of a mixture of active substances on plastic mulches. Previously, eight vegetable farmers in the region of Murcia (Southeast Spain) were interviewed to discover which types of pesticides and plastic mulches were commonly used in the research area. All interviewed farmers used either LDPE, PAC or Bio plastic mulch in their crop production. All farmers used similar vegetable rotations and the type of plastic mulch used was not linked to the type of crop. We were able to assemble a full list of the active substances in the pesticides that were used by the farmers. Some active substances on the list were not analyzed due to logistical and financial limitations. The final list of 38 active substances from 17 insecticides, 15 fungicides and 6 herbicides is presented in supplementary Table S3. 
Three plastic mulches: LDPE, PAC and Bio, were incubated with or without active substances. Additionally, one control treatment containing a mixture of the active substances without plastic was also tested. Therefore, in total, seven treatments were set up in glass tubes (Table 1). All treatments were carried out in duplicate. Each tube contained $5 \mathrm{~mL}$ of a solution (either with or without the mixture of active substances) and a piece of $3 \times 3 \mathrm{~cm}^{2}$ plastic mulch, depending on the treatment (Figure 1). Therefore, we can distinguish two phases: the plastic mulch and the incubation solution.

\section{Plastic mulch types used in the experiment.}

Samples of unused plastic mulch were collected from farmers' warehouses located in the region of Murcia for each of the mulches: LDPE, PAC and Bio. All three types of mulches were black. The detailed composition of the plastic was not given by the producers since it was protected by intellectual property regulations. In addition to the main polymer, all plastics contained additives used to control the color, elasticity and resistance of the mulch (Crawford et al. 2017a, Sintim and Flury 2017). The LDPE mulch came from Reyenvas (Spain). LDPE plastic mulch is designed to be resistant and removed after the harvest. The PAC mulch (commercial name "actiblack") came from Trioplast SMS SAS (France). PAC mulch is made of LDPE with the addition of a pro-oxidant additive that increases its decay such that farmers usually incorporate it into the soil after harvest instead of removing it. Finally, Bio mulch (commercial name "Sotrafilm Black Biodegradable") was bought from Sotrafa (Spain). The available information states that it is a "biopolymer film made with biodegradable and renewable raw materials and particular carbon black content to get an optimum opacity for mulching use" (Sotrafa 2018). The compliance with the biodegradable plastic mulch norms EN 17033:2018 (CEN 2018) or ISO 17556:2019 (ISO 2019) was not specified. The composition of the biodegradable mulch was investigated using the Varian 1000 FTIR (Fourier transform infrared) spectrometer from the Aquatic Ecology and Water Quality Management group of Wageningen University. Eight scans were performed for the background and the samples. The spectrometer produced spectra ranging from $3750 \mathrm{~cm}^{-1}$ to $400 \mathrm{~cm}^{-1}$ with a resolution of $4 \mathrm{~cm}^{-1}$. The comparison of the spectra with polymer libraries (HR Hummel Polymer and Additives, HR Spectra Polymers and Plasticizers by ATR, HR Sprouse Polymers by Transmission) gave high percentages of match for Polyester terephthalic acid (78\% match), Polybutylene terephthalate (72\% match) and polyethylene terephthalate (65.6\% match) (Figure S1, Table S1). Therefore, the biodegradable plastic mulch may have been composed of Polybutylene terephthalate, polyethylene terephthalate or other similar copolyester of terephthalic acid. It is most likely that the main polymer was Polybutylene adipate terephthalate as it is a copolyester of terephthalic acid commonly used for its biodegradability properties (Weng et al. 2013).

The pieces of plastic mulch that were collected were manually cut into $3 \times 3 \mathrm{~cm}^{2}$ pieces before incubation. The $3 \times 3 \mathrm{~cm}^{2}$ pieces of mulch were manipulated so that they fit into the glass tubes but they were not folded (Figure 1). Plastic pieces were fully immersed in the solution so that the incubation solution was in contact with the whole surface of the piece of plastic.

\section{Incubation solution and incubation conditions.}

For each of the 38 active substances (Table S3), the Pesticide Properties Database (BPDB 2018, PPDB 2018) was used to get the molar mass, the aqueous hydrolysis half-life time at $20^{\circ} \mathrm{C}$ and $\mathrm{pH} 7$ (DT50; indicator of degradation in water), the solubility in water at $20^{\circ} \mathrm{C}$ and the 
181

182

183

184

185

186

187

188

189

190

191

192

193

194

195

196

197

198

199

200

201

202

203

204

205

206

207

208

209

210

211

212

213

214

215

216

217

218

219

220

221

222

223

224

225

226

octanol-water partition coefficient at $\mathrm{pH} 7,20^{\circ} \mathrm{C}(\log \mathrm{P})$. The octanol-water partition coefficient $(\log \mathrm{P})$ was used as a measure of the active substances hydrophobicity, which plays a key role in sorption (Leo et al. 1971). A concentration of $1000 \mathrm{ng} \cdot \mathrm{mL}^{-1}$ of each active substance was mixed in a solution of $10 \%$ acetonitrile and $90 \%$ distilled water so that there was $5000 \mathrm{ng}$ of each active substance in the final volume of $5 \mathrm{~mL}$. The concentration was the same as in Nerin et al (1996) and the mass of pesticides available per area of plastic was similar. Acetonitrile in the incubation solution may have helped the dissolution of hydrophobic active substances since the solubility in water for some of the substances was low (Table S3). The initial presence of active substances in the plastic was assessed using the same incubation solution $(90 \%$ distilled water $+10 \%$ acetonitrile) without active substances applied (Treatments LDPE+W, PAC $+\mathrm{W}, \mathrm{Bio}+\mathrm{W}$ ).

All glass tubes were incubated at $35^{\circ} \mathrm{C}$ for 15 days in a laboratory oven. The temperature was representative of the temperature under the plastic mulch in semi-arid regions (Nerín et al. 1996). Tubes were kept in the dark, without additional stirring or oxygenation during the 15 days. A period of 15 days was enough time to reach the sorption equilibrium, as reported on LDPE films by Nerin et al (1996) and allowed us to study the degradation of the substances.

\section{Active substance extraction and determination.}

After incubation, the plastic pieces were carefully washed with distilled water, cut into $5 \times 5 \mathrm{~mm}^{2}$ pieces and transferred to a $50 \mathrm{~mL}$ tube for active substance extraction. The extraction method was adapted from Nerin et al (1996) and the QuEChERS approach (Anastassiades et al. (2003). Nerin et al, (1996) showed with a similar extraction method that a single extraction was sufficient for a quantification. The analytical method was similar to the one described in Mol et al. (2008) and Silva et al. (2018). Plastic tubes and plastic vials were used for extraction and quantification procedures. Given the concentration of acetonitrile and the short time of extraction, we do not expect significant losses of active substances based on the quality assessment method of Mol et al. (2008) and Silva et al. (2018). All plastic samples were spiked with ${ }^{13} \mathrm{C}$-caffeine (used as internal standard to assess the procedure efficiency), and mixed with 5 $\mathrm{mL}$ of distilled water and $10 \mathrm{~mL}$ of acetonitrile containing 1\% acetic acid (Mol et al. 2008). Tubes were exposed to an ultrasonic bath for one hour and agitated end-over-end for another hour. Then, $1 \mathrm{~g}$ of sodium acetate and $4 \mathrm{~g}$ of magnesium sulphate were added to induce phase separation. After centrifugation, $250 \mu \mathrm{L}$ of the supernatant (acetonitrile phase) was collected, mixed with $250 \mu \mathrm{L}$ of distilled water and filtered in a filter vial for analysis. The incubation solution was taken from the test tube and diluted 40 times in a solution of acetonitrile $+1 \%$ acetic acid and distilled water $(1: 1)$.

The active substance content was analyzed with a liquid chromatography-tandem mass spectrometry (LC-MS/MS) system (Silva et al. 2018a) with mobile phases of $0.1 \%$ formic acid and $5 \mathrm{mM}$ ammonium formate in water (eluent A) or in $95 \%$ methanol, 5\% water (eluent B). The gradient used to elute all compounds from the column is shown in Table S2. LC-MS/MS measurements were performed on a Xevo TQ-S (tandem quadrupole mass spectrometer) system coupled with an Acquity UPLC (ultra-performance liquid chromatography) system, both from Waters (Milford, MA, USA). Each LC-MS/MS analysis included a calibration curve of nine fortified blanks $\left(0,0.125,0.25,0.5,1,2.5,5,10,25 \mathrm{ng} \cdot \mathrm{mL}^{-1}\right)$ in a solution of acetonitrile $+1 \%$ acetic acid and distilled water (1:1). The extraction procedure performed on the plastic not incubated with pesticides provided a matrix extract. A standard in matrix was prepared from the matrix extract fortified at $5 \mathrm{ng} \cdot \mathrm{mL}^{-1}$ and injected after every 10 sample measurements. The software MassLynx ${ }^{\mathrm{TM}}$ (Version 4.1, Waters) was used to collect the data and integrate the peaks.

Peer] reviewing PDF | (2020:02:46097:2:1:NEW 6 Aug 2020) 
227

228

229

230

231

232

233

234

235

236

237

238

239

240

241

242

243

244

245

246

247

248

249

250

251

252

253

254

255

256

257

258

259

260

261

262

263

264

265

266

267

268

269

270

A limit of quantification (LOQ) was calculated for each compound according to the lowest calibration level inside the linearity range (deviation of back-calculated concentration from true concentration within $\pm 20 \%$ ) and an ion ratio within $\pm 30 \%$ of the average of calibration (European Commission 2017) (Table S4). The active substance contents below the LOQ were considered to be zero during data processing.

\section{Data processing.}

Data calculation and plotting were done in $\mathrm{R}$ version 3.4.2. Calculations were performed using the mean of the duplicate treatments. The percentage of sorption was calculated for each tube containing the plastic mulch and the active substances mixture as the ratio between the mass of active substances detected in the plastic extract and the mass of the active substances added. The sorption calculation did not take into account the possible decay of active substances in the solution or the incomplete extraction of sorbed substances. As a consequence, calculated sorption percentages may have been lower than the real sorption of active substances on plastic mulches. The Shapiro-Wilks test was performed at $\mathrm{p}<0.05$ to check the normality of the percentage of sorption with the function shapiro.test of $\mathrm{R}$. Then percentages of sorption were compared among the different plastic types using a one-way non-parametric ANOVA (Kruskal-Wallis comparison at $\mathrm{p}<0.05)$ with functions kruskal.test and dunnTest in R. Given p-values were adjusted using the Benjamini-Hochberg method.

The recovery ratio (sum of the mass of active substances measured in all compartments divided by the mass of active substances added) was calculated for each treatment where active substances were added. The difference between the mass recovered and the mass added was considered the mass of active substances decayed during the incubation. The decay calculated for active substances without plastic, minus the decay calculated in the presence of plastic, gave an estimation of the decay reduction in the case of active substance sorption.

The $l m$ function of $\mathrm{R}$ calculated the linear coefficients, the coefficient of determination $\left(\mathrm{R}^{2}\right)$ and the $\mathrm{p}$-value of linear regressions between percentage of sorption and $\log \mathrm{P}$, as well as between decay reduction and percentage of sorption.

\section{Results}

\section{Active substance sorption on plastic mulch.}

The mean sorption rate of all active substances on each type of plastic are shown in Figure 2. The measured sorption rates did not follow a normal distribution (Shapiro-Wilks test, $p$ $<0.001)$. LDPE and PAC plastics showed no significant differences for sorption of active substances $(\mathrm{p}>0.05)$, with an average of $\sim 23 \%$. Bio mulch showed a significantly higher sorption rate than LDPE and PAC mulches $(p<0.05)$ with an average value of $\sim 50 \%$. In fact, 20 out of the 38 tested compounds showed a sorption rate $>50 \%$ on Bio, whereas only 9 and 7 had a sorption rate $>50 \%$ on LDPE and PAC mulches, respectively.

Sorption rates $(\%)$ of each active substance to the different plastic types (treatments $\mathrm{LDPE}+\mathrm{P}, \mathrm{PAC}+\mathrm{P}$ and Bio $+\mathrm{P})$ are presented in Figure 3 and in Table S4. We observed that 14 compounds $(37 \%)$ had a sorption rate $>10 \%$ on all plastic mulches. Ten compounds had a sorption rate $<1 \%$ on all plastics. Three compounds, Chlorpyrifos, Oxyfluorfen and Pendimethalin, had a sorption rate $>80 \%$ on all plastics. 
271

272

273

274

275

276

277

278

279

280

281

282

283

284

285

286

287

288

289

290

291

292

293

294

295

296

297

298

299

300

301

302

303

304

305

306

307

308

309

310

Active substances with higher log $\mathrm{P}$ values tended to show higher sorption rates. In fact, the sorption rate was positively correlated with $\log \mathrm{P}\left(\mathrm{R}^{2}=0.96, \mathrm{p}<0.001\right)$ (Figure 4). Nevertheless, it is worth noting that active substances with the same log $\mathrm{P}$ and the same plastic type could have had significantly different sorption rates (e.g. Figure $3 ; \log \mathrm{P}($ Chlorpyrifos) $=$ $\log \mathrm{P}($ Cyflufenamid $)=4.7$ whereas mean sorption on LDPE was $88 \%$ for Chlorpyrifos and $45 \%$ for Cyflufenamid).

For the plastic mulch with no added active substances (LDPE+W, PAC $+\mathrm{W}$ and $\mathrm{Bio}+\mathrm{W})$, no active substances were found in the PAC or Bio extracts but low levels (maximum: 16 ng; average: $7.7 \mathrm{ng}$ ) were found in the samples with LDPE in one duplicate but not in the other duplicate. These very low levels (compared to the $5000 \mathrm{ng}$ of active substance added) are likely to have come from contamination in the liquid chromatography column.

For active substance samples where no plastic was added, half of the compounds had a recovery ratio $<50 \%$ and 29 compounds $(75 \%)$ had a recovery ratio $<90 \%$ (Figure S2). It is worth noting that without plastic, active substances with higher $\log \mathrm{P}$ tended to have lower recovery. Moreover, active substances with a shorter DT50 in water tended to have a lower recovery ratio; meaning that lower recovery is likely to be explained by higher decay during incubation. In the next section, we assumed that the missing percentage (1-recovery) was due to the degradation of the active substances during incubation.

\section{Decay reduction due to sorption of active substances.}

The sorption of active substances significantly reduced their decay in comparison to the active substances without plastic mulch (Figure 5). The estimated decay for active substances with sorption $>80 \%$ was $\sim 70 \%$ lower than the decay without plastics. We measured a decay reduction of $\sim 27 \%$ for LDPE and PAC and $\sim 37 \%$ for Bio for active substances with a sorption $>$ $0.01 \%$. The decay reduction showed a significant linear relationship with the percentage of sorption $\left(\mathrm{R}^{2}=0.90, \mathrm{p}<0.001\right)$. The greater sorption on Bio directly reflected on a greater decay reduction for active substances.

\section{Discussion}

We did a single point sorption experiment. Based on previous studies, 15 days of sorption at $35^{\circ} \mathrm{C}$ were enough to reach steady states (Nerín et al. 1996, Sharom and Solomon 1981). Kinetic sorption experiments would be needed to calculate sorption coefficients. The active substance extraction procedure was partly based on Nerín et al. (1996) and was not tested again. In case of a low extraction rate, we underestimated the sorption on plastic and over-estimated the decay of active substance incubated with plastic. We don't expect the extraction rate to perform differently for LDPE, PAC or Bio. Therefore, a low extraction rate would not change our conclusion.

The Bio mulch may have contained polybutylene adipate terephthalate and Bio mulch had a higher sorption than LDPE and PAC mulches. Higher sorption may be related to the 
311 chemical properties of the polymer used or to the specific surface area of the mulch (Aslam et al. 312 2013). Polybutylene adipate terephthalate may have a better affinity with the active substances

313 than the LDPE because of aromatic-interactions and potential hydrogen bonds when the polymer 314 is altered (Palsikowski et al. 2018). Aged polybutylene adipate terephthalate is able to form

315 hydrogen bonds with organic chemicals (Weng et al. 2013). It is possible that the 15 day 316 incubation at $35^{\circ} \mathrm{C}$ caused an alteration in the Bio mulch and increased the formation of 317 hydrogen bonds between the Bio mulch and the active substances. Additionally, biodegradable 318 mulches tend to be made with smaller polymer fiber diameters that increase its specific surface 319 area (Chinaglia et al. 2018, Hayes et al. 2012) and its biodegradation (Brodhagen et al. 2015, Chinaglia et al. 2018). A greater specific surface area would also increase the sorption (Liu et al. 2019).

Our study showed that for 20 compounds sorption on Bio mulch was higher than $50 \%$. Sorbed active substances are likely to alter the degradation of the Bio mulch by affecting the soil microbiome (Oyeleke and Oyewole 2019). According to the International Organization for Standardization (ISO) 17556, biodegradable mulch should reach at least $90 \%$ biodegradation in the soil within two years (Carol et al. 2017). A field study showed that after 397 days in the soil, three different kinds of biodegradable mulches (Crown 1, BioAgri and SB-PLA-11) had various deterioration rates $(100 \%, 65 \%$ and very little deterioration, respectively) (Jeremy et al. 2013). Plastic degradation studies should take into account that pesticides are likely to be sorbed on plastic and may reduce its biodegradation. On the other hand, the efficiency of pesticides in the soil (i.e. herbicides, fungicides) depends on their availability. Therefore, plastic mulch may decrease the efficiency of pesticides by decreasing their release into the soil when plastic mulch debris accumulates and the pesticides are sprayed on the soil. The aging of plastics (Liu et al. 2019) and the pesticide sorption in soils contaminated with plastics (Hüffer et al. 2019), for different soil types and organic matter contents, are factors that need to be studied to understand the interactions between plastic debris and pesticide residues.

LDPE and PAC mulches have similar sorption and their composition only differs in the additives that are added to them. Thus, the additives present in PAC mulch do not seem to change the sorption property of the original LDPE polymer. The sorption was much higher for the Bio mulch. As a consequence, knowing the exact chemical formulation of the polymers used to make plastic mulches and the specific surface area of the mulch are essential in understanding the mechanisms of the sorption of pesticides on plastic. Better, cheaper and faster analysis of plastic composition (Corradini et al. 2019, Mintenig et al. 2017) or regulations forcing producers to share the chemical formulation of polymers could help filling this knowledge gap.

The sorption on plastic varied for all active substances, being higher for those with higher $\log$ P. In fact, $\log$ P, as a measure of hydrophobicity (Leo et al. 1971), plays a key role in sorption processes (Aslam et al. 2013). Nevertheless, the log P did not predict the sorption for all active substances so mechanisms other than hydrophobicity must play a role (Guo et al. 2000). Some active substances may have had an impact on the sorption of some other ones, likely due to chemical interactions between them. Interactions between active substances might have changed 
351 the active substance degradation in solution as well as in the plastic matrix. These results

352 highlight the need for more detailed studies to understand the mechanisms of pesticide sorption

353 on plastic.

354 The highest degradation rates were obtained for active substances with low stability to

355 hydrolysis and low stability in aqueous solution (aqueous hydrolysis DT50 (days) at 20 ${ }^{\circ} \mathrm{C}$, pH 7

356 and degradation in water DT50 (days) (PPDB 2018)). We can assume that the pesticide

357 degradation in the glass tube was mainly due to hydrolysis (Fenner et al. 2013). Volatilization in

358 the gaseous phase in the tube or incomplete solubilization could have played a role in the

359 estimation of the decay. However, neither the decay reduction nor the sorption was correlated

360 with the solubility, meaning that hydrolysis was the most likely process leading to the

361 degradation. The decay of active substances from pesticides was reduced by sorption. It is

362 commonly accepted that sorption limits pesticide degradation by reducing its partitioning into the

363 liquid phase (Guerin and Boyd 1997, O'Loughlin et al. 2000). Additionally, soil microorganisms

364 degrade preferably or exclusively chemicals that are present in the soil solution (Boivin, et al.

365 2005a). Thus, sorbed active substances would undergo less degradation by microorganisms

366 (Liang et al. 2011). Since plastic debris could be transported by wind and water (Liu et al. 2014),

367 pesticide transport (Teuten et al. 2007) and degradation models (Silva et al. 2018b) should take

368 into account the sorption of pesticides on plastic (Villeneuve et al. 1988).

369

370

The applied experimental design was based on Nerin et al. (1996) to reveal a potential of commonly used active substances from pesticides to be sorbed and protected from decay on conventional (LDPE) and new (PAC and Bio) plastic mulches. The sorption condition applied

372 here does not reflect real conditions in fields. The 38 active substances were applied together at

373 the same concentration, in the same solution. We can assume then that most hydrophobic active substances may have reduced the sorption of the rest of the substances due to the competitive sorption among all active substances. Competitive sorption would occur to a negligible extent in the field because fewer active substances would be applied simultaneously and the use of pesticides would be spread over the whole growing period resulting in seasonal variations. Moreover, the applied concentration of $1000 \mathrm{ng} \cdot \mathrm{mL}^{-1}$ exceeded the solubility in water for some compounds. A non-dissolved fraction of the active substances may had formed a stock within the liquid medium. The incubation was done in glass tubes, in the dark, at $35^{\circ} \mathrm{C}$ without temperature variation, stirring or oxygenation. In the field, active substances could undergo sorption on soil particles, degradation by light and by microorganisms or volatilization. Additionally, the presence of $10 \%$ acetonitrile in the incubation solution could have reduced the sorption of most hydrophobic contaminants in plastics and polymers (Teuten et al. 2007). Sorption percentages on plastic may be higher without acetonitrile and with less competitive sorption. However, sorption percentages could be reduced by additional degradation processes (Fenner et al. 2013, Kumar et al. 2018), volatilization and sorption to soil particles (Boivin et al. 2005). Finally, plastic degradation may change the chemical properties of the polymer or the specific surface area of the mulch (Hayes et al. 2017, Liu et al. 2019) and change the sorption of active substances (Aslam et al. 2013). Despite these last issues comparing the conditions of this experiment with 
391

392

393

394

395

396

397

398

399

400

401

402

403

404

405

406

407

408

409

410

411

412

413

414

415

416

417

418

419

420

421

422

423

424

425

426

427

428

429

430

431

432

actual conditions in the field, our study highlights the need for further research on plastic mulchpesticide systems since there is a real interaction between both components, which could negatively affect pesticides and plastic degradation in the field. Thus, it is essential to address these topics under field conditions (Yang et al. 2018) taking into account new and aged plastics (Liu et al. 2019).

The sorption of active substances on plastic may change the toxicity of both the pesticides and the plastic. In fact, if plastic debris is ingested by organisms (Colabuono et al. 2010, Huerta Lwanga et al. 2017) then the sorbed active substances could potentially be desorbed in organisms (Teuten et al. 2007). On the other hand, the sorption to plastic may reduce the bioavailability of active substances, especially reducing the peak concentration after pesticides application. The reduced exposure of soil organisms could be beneficial for the ecosystem. Contaminated plastics may as well release active substances in the soil solution and contribute to plastic toxicity (Machado et al. 2018, Qi et al. 2018). In a similar way, active substances sorbed on plastic may decrease the plastic's degradation by soil organisms because of the toxicity of the active substances. Active substance sorption is of particular concern when it comes to the degradation of biodegradable mulch since the degradation of biodegradable mulch relies heavily on the activity of microorganisms in the soil. As a consequence, further studies are needed to assess the degradation of plastic debris, especially biodegradable plastics in soils where pesticides are sprayed.

\section{Conclusions}

This study reveals that sorption of active substances on plastic depends on both the chemical structure of the active substance and the type of plastic mulch. Sorption was higher for active substances with higher $\log \mathrm{P}$ (octanol-water partition coefficient) and although it was similar between LDPE and PAC plastics, it was significantly higher on Bio mulch. Moreover, sorption of active substances on plastic reduced the decay of active substances. Therefore, the sorption of active substances can change the eco-toxicity and decay of both the active substances and the plastic debris. The sorption can also affect the transport pattern of active substances, especially when biodegradable plastic is used. More research is needed to evaluate the dynamics and consequences of the sorption of active substances from pesticides on plastic mulches in environmental conditions. With more research, scientists can propose guidelines for the use of plastic mulches in agro-ecosystems in order to avoid soil and water pollution.

\section{Acknowledgements}

We are thankful for the contribution of the farmers from the region of Murcia, Spain. We would like to thank Klaas Oostindie for his graphic support and Robin Palmer for the language editing. We are very grateful to Frits Gillissen from the Aquatic Ecology and Water Quality Management group of Wageningen University \& Research for the FTIR analysis of the plastic 
433 mulch. We would also like to thank Esperanza Huerta Lwanga for her invaluable comments and 434 revisions.

435

436

\section{References}

437

438

439

440

441

442

443

444

445

446

447

448

449

450

451

452

453

454

455

456

457

458

459

460

461

462

463

464

465

466

467

468

469

470

471

472

473

474

475

476

477

478

479

480

481

Ahmed, T., Shahid, M., Azeem, F., Rasul, I., Shah, A., Noman, M., Hameed, A., Manzoor, N., Manzoor, D.I. and Muhammad, S. (2018) Biodegradation of plastics: current scenario and future prospects for environmental safety.

Anastassiades, M., Lehotay, S.J., Stajnbaher, D. and Schenck, F.J. (2003) Fast and easy multiresidue method employing acetonitrile extraction/partitioning and "dispersive solid-phase extraction" for the determination of pesticide residues in produce. J AOAC Int 86(2), 412-431. Aslam, S., Garnier, P., Rumpel, C., Parent, S.E. and Benoit, P. (2013) Adsorption and desorption behavior of selected pesticides as influenced by decomposition of maize mulch. Chemosphere 91(11), 1447-1455.

Boivin, A., Cherrier, R. and Schiavon, M. (2005) A comparison of five pesticides adsorption and desorption processes in thirteen contrasting field soils. Chemosphere 61(5), 668-676. BPDB (2018) Bio-Pesticides DataBase.

Briassoulis, D. (2004) An Overview on the Mechanical Behaviour of Biodegradable Agricultural Films.

Brodhagen, M., Peyron, M., Miles, C. and Inglis, D.A. (2015) Biodegradable plastic agricultural mulches and key features of microbial degradation. Applied Microbiology and Biotechnology 99(3), 1039-1056.

Carol, M., Lisa, D., Shuresh, G. and Douglas, G.H. (2017) Suitability of Biodegradable Plastic Mulches for Organic and Sustainable Agricultural Production Systems. HortScience horts 52(1), 10-15.

CEN (2018) EN 17033:2018 Plastics - Biodegradable mulch films for use in agriculture and horticulture - Requirements and test methods.

Chinaglia, S., Tosin, M. and Degli-Innocenti, F. (2018) Biodegradation rate of biodegradable plastics at molecular level. Polymer Degradation and Stability 147, 237-244.

Colabuono, F.I., Taniguchi, S. and Montone, R.C. (2010) Polychlorinated biphenyls and organochlorine pesticides in plastics ingested by seabirds. Marine Pollution Bulletin 60(4), 630634.

Corradini, F., Bartholomeus, H., Huerta Lwanga, E., Gertsen, H. and Geissen, V. (2019) Predicting soil microplastic concentration using vis-NIR spectroscopy. Science of The Total Environment 650, 922-932.

Crawford, C.B., Quinn, B., Crawford, C.B. and Quinn, B. (2017a) Microplastic Pollutants, pp. 57100, Elsevier.

Crawford, C.B., Quinn, B., Crawford, C.B. and Quinn, B. (2017b) Microplastic Pollutants, pp. 131-157, Elsevier.

European Commission (2016) Proposal for a Regulation of the European Parliament and of the Council laying down rules on the making available on the market of CE marked fertilising products and amending Regulations, European Commission, Eur-Lex.

European Commission (2017) Guidance document on analytical quality control and method validation procedures for pesticide residues and analysis in food and feed. safety, D.g.f.h.a.f. (ed), Supercedes.

Fenner, K., Canonica, S., Wackett, L.P. and Elsner, M. (2013) Evaluating Pesticide Degradation in the Environment: Blind Spots and Emerging Opportunities. Science 341(6147), 752. G. Hayes, D., Dharmalingam, S., C. Wadsworth, L., Leonas, K., Miles, C. and A. Inglis, D. (2012) Biodegradable Agricultural Mulches Derived from Biopolymers.

Peer] reviewing PDF | (2020:02:46097:2:1:NEW 6 Aug 2020) 
482 Guerin, W.F. and Boyd, S.A. (1997) Bioavailability of naphthalene associated with natural and

483

484

485

486

487

488

489

490

491

492

493

494

495

496

497

498

499

500

501

502

503

504

505

506

507

508

509

510

511

512

513

514

515

516

517

518

519

520

521

522

523

524

525

526

527

528

529

530

531

synthetic sorbents. Water Research 31(6), 1504-1512.

Guo, L., Jury, W.A., Wagenet, R.J. and Flury, M. (2000) Dependence of pesticide degradation on sorption: nonequilibrium model and application to soil reactors. Journal of Contaminant Hydrology 43(1), 45-62.

Hayes, D.G., Wadsworth, L.C., Sintim, H.Y., Flury, M., English, M., Schaeffer, S. and Saxton, A.M. (2017) Effect of diverse weathering conditions on the physicochemical properties of biodegradable plastic mulches. Polymer Testing 62, 454-467.

Hirai, H., Takada, H., Ogata, Y., Yamashita, R., Mizukawa, K., Saha, M., Kwan, C., Moore, C., Gray, H., Laursen, D., Zettler, E.R., Farrington, J.W., Reddy, C.M., Peacock, E.E. and Ward, M.W. (2011) Organic micropollutants in marine plastics debris from the open ocean and remote and urban beaches. Marine Pollution Bulletin 62(8), 1683-1692.

Hogg, D.G., Adrian; Hann, Simon; Ettlinger, Sarah (2016) The impact of the use of "oxodegradable" plastic on the environment. Environment, E.C.D.-G.f. (ed).

Huerta Lwanga, E., Gertsen, H., Gooren, H., Peters, P., Salánki, T., van der Ploeg, M., Besseling, E., Koelmans, A.A. and Geissen, V. (2017) Incorporation of microplastics from litter into burrows of Lumbricus terrestris. Environmental Pollution 220, 523-531.

Hüffer, T., Metzelder, F., Sigmund, G., Slawek, S., Schmidt, T.C. and Hofmann, T. (2019)

Polyethylene microplastics influence the transport of organic contaminants in soil. Science of The Total Environment 657, 242-247.

ISO (2019) ISO 17556:2019 Plastics - Determination of the ultimate aerobic biodegradability of plastic materials in soil by measuring the oxygen demand in a respirometer or the amount of carbon dioxide evolved, p. 26.

Jeremy, S.C., Debra, A.I. and Carol, A.M. (2013) Deterioration of Three Potentially Biodegradable Plastic Mulches Before and After Soil Incorporation in a Broccoli Field Production System in Northwestern Washington. HortTechnology hortte 23(6), 849-858.

Jiang, R., Li, X., Zhou, M., Li, H.J., Zhao, Y., Yi, J., Cui, L.L., Li, M., Zhang, J.G. and Qu, D. (2016) Plastic film mulching on soil water and maize (Zea mays L.) yield in a ridge cultivation system on Loess Plateau of China. Soil Science and Plant Nutrition 62(1), 1-12.

Kasirajan, S. and Ngouajio, M. (2012) Polyethylene and biodegradable mulches for agricultural applications: a review. Agronomy for Sustainable Development 32(2), 501-529.

Kijchavengkul, T. and Auras, R. (2008) Compostability of polymers. Polymer International 57(6), 793-804.

Kumar, S., Kaushik, G., Dar, M.A., Nimesh, S., LÓPez-Chuken, U.J. and Villarreal-Chiu, J.F. (2018) Microbial Degradation of Organophosphate Pesticides: A Review. Pedosphere 28(2), 190-208.

Lee, H., Shim, W.J. and Kwon, J.-H. (2014) Sorption capacity of plastic debris for hydrophobic organic chemicals. Science of The Total Environment 470-471, 1545-1552.

Leo, A., Hansch, C. and Elkins, D. (1971) Partition coefficients and their uses. Chemical Reviews 71(6), 525-616.

Liang, B., Yang, C., Gong, M., Zhao, Y., Zhang, J., Zhu, C., Jiang, J. and Li, S. (2011) Adsorption and degradation of triazophos, chlorpyrifos and their main hydrolytic metabolites in paddy soil from Chaohu Lake, China. Journal of Environmental Management 92(9), 2229-2234. Liu, E.K., He, W.Q. and Yan, C.R. (2014) 'White revolution' to 'white pollution'-agricultural plastic film mulch in China. Environmental Research Letters 9(9), 091001.

Liu, G., Zhu, Z., Yang, Y., Sun, Y., Yu, F. and Ma, J. (2019) Sorption behavior and mechanism of hydrophilic organic chemicals to virgin and aged microplastics in freshwater and seawater. Environmental Pollution 246, 26-33.

Machado, A.A.S., Kloas, W., Zarfl, C., Hempel, S. and Rillig, M.C. (2018) Microplastics as an emerging threat to terrestrial ecosystems. Global Change Biology 24(4), 1405-1416.

Peer] reviewing PDF | (2020:02:46097:2:1:NEW 6 Aug 2020) 
532 Mato, Y., Isobe, T., Takada, H., Kanehiro, H., Ohtake, C. and Kaminuma, T. (2001) Plastic

533 Resin Pellets as a Transport Medium for Toxic Chemicals in the Marine Environment.

534 Environmental Science \& Technology 35(2), 318-324.

535 Mintenig, S.M., Int-Veen, I., Löder, M.G.J., Primpke, S. and Gerdts, G. (2017) Identification of

536 microplastic in effluents of waste water treatment plants using focal plane array-based micro-

537 Fourier-transform infrared imaging. Water Research 108, 365-372.

538 Mol, H.G.J., Plaza-Bolaños, P., Zomer, P., de Rijk, T.C., Stolker, A.A.M. and Mulder, P.P.J.

539 (2008) Toward a Generic Extraction Method for Simultaneous Determination of Pesticides,

540 Mycotoxins, Plant Toxins, and Veterinary Drugs in Feed and Food Matrixes. Analytical

541 Chemistry 80(24), 9450-9459.

542 Nerín, C., Tornés, A.R., Domeño, C. and Cacho, J. (1996) Absorption of Pesticides on Plastic

543 Films Used as Agricultural Soil Covers. Journal of Agricultural and Food Chemistry 44(12),

544 4009-4014.

545 O'Loughlin, E.J., Traina, S.J. and Sims, G.K. (2000) Effects of sorption on the biodegradation of

546 2-methylpyridine in aqueous suspensions of reference clay minerals. Environmental Toxicology

547 and Chemistry 19(9), 2168-2174.

548 Oever, M.v.d., Molenveld, K., Zee, M.v.d., Bos, H. and tte (2017) Bio-based and biodegradable

549 plastics : facts and figures : focus on food packaging in the Netherlands, Wageningen Food \&

550 Biobased Research, Wageningen.

551 Oyeleke, S.B. and Oyewole, O. (2019) Effect of herbicide (pendimethalin) on soil microbial

552 population.

553 Palsikowski, P.A., Kuchnier, C.N., Pinheiro, I.F. and Morales, A.R. (2018) Biodegradation in Soil

554

555

556

557

558

559

560

561

562

563

564

565

566

567

568

569

570

571

572

573

574

575

576

577

578 of PLA/PBAT Blends Compatibilized with Chain Extender. Journal of Polymers and the Environment 26(1), 330-341.

PPDB (2018) Pesticide Properties DataBase

Qi, Y., Yang, X., Pelaez, A.M., Huerta Lwanga, E., Beriot, N., Gertsen, H., Garbeva, P. and Geissen, V. (2018) Macro- and micro- plastics in soil-plant system: Effects of plastic mulch film residues on wheat (Triticum aestivum) growth. Science of The Total Environment 645, 10481056.

Ramos, L., Berenstein, G., Hughes, E.A., Zalts, A. and Montserrat, J.M. (2015) Polyethylene film incorporation into the horticultural soil of small periurban production units in Argentina. Science of The Total Environment 523, 74-81.

Rillig, M.C. (2012) Microplastic in Terrestrial Ecosystems and the Soil? Environmental Science \& Technology 46(12), 6453-6454.

Sander, M. (2019) Biodegradation of Polymeric Mulch Films in Agricultural Soils: Concepts, Knowledge Gaps, and Future Research Directions.

Seidensticker, S., Grathwohl, P., Lamprecht, J. and Zarfl, C. (2018) A combined experimental and modeling study to evaluate $\mathrm{pH}$-dependent sorption of polar and non-polar compounds to polyethylene and polystyrene microplastics. Environmental Sciences Europe 30(1), 30.

Selke, S., Auras, R., Nguyen, T.A., Castro Aguirre, E., Cheruvathur, R. and Liu, Y. (2015) Evaluation of Biodegradation-Promoting Additives for Plastics. Environmental Science \& Technology 49(6), 3769-3777.

Sharom, M.S. and Solomon, K.R. (1981) Adsorption and Desorption of Permethrin and Other Pesticides on Glass and Plastic Materials used in Bioassay Procedures. Canadian Journal of Fisheries and Aquatic Sciences 38(2), 199-204.

Shen, L., Worrell, E. and Patel, M. (2010) Present and future development in plastics from biomass. Biofuels, Bioproducts and Biorefining 4(1), 25-40.

579 Silva, V., Mol, H.G.J., Zomer, P., Tienstra, M., Ritsema, C.J. and Geissen, V. (2018a) Pesticide

580 residues in European agricultural soils - A hidden reality unfolded. Science of The Total

581 Environment. 
582 Silva, V., Montanarella, L., Jones, A., Fernández-Ugalde, O., Mol, H.G.J., Ritsema, C.J. and 583 Geissen, V. (2018b) Distribution of glyphosate and aminomethylphosphonic acid (AMPA) in agricultural topsoils of the European Union. Science of The Total Environment 621, 1352-1359. Sintim, H.Y. and Flury, M. (2017) Is Biodegradable Plastic Mulch the Solution to Agriculture's Plastic Problem? Environmental Science \& Technology 51(3), 1068-1069.

587 Sotrafa (2018) Sotrafilm Black Biodegradable. Steinmetz, Z., Wollmann, C., Schaefer, M., Buchmann, C., David, J., Tröger, J., Muñoz, K., Frör, O. and Schaumann, G.E. (2016) Plastic mulching in agriculture. Trading short-term agronomic benefits for long-term soil degradation? Science of The Total Environment 550, 690705.

592 Teuten, E.L., Rowland, S.J., Galloway, T.S. and Thompson, R.C. (2007) Potential for Plastics to 593 Transport Hydrophobic Contaminants. Environmental Science \& Technology 41(22), 77595947764.

595 van der Meulen, E.S., Nol, L. and Cammeraat, L.H. (2006) Effects of Irrigation and Plastic Mulch 596 on Soil Properties on Semiarid Abandoned Fields. Soil Science Society of America Journal 597 70(3), 930-939.

598 Villeneuve, J.-P., Lafrance, P., Banton, O., Frechette, P. and Robert, C. (1988) A sensitivity 599 analysis of adsorption and degradation parameters in the modeling of pesticide transport in 600 soils. Journal of Contaminant Hydrology 3(1), 77-96.

601 Weng, Y.-X., Jin, Y.-J., Meng, Q.-Y., Wang, L., Zhang, M. and Wang, Y.-Z. (2013)

602 Biodegradation behavior of poly(butylene adipate-co-terephthalate) (PBAT), poly(lactic acid)

603 (PLA), and their blend under soil conditions. Polymer Testing 32(5), 918-926.

604 Yang, X., Bento, C.P.M., Chen, H., Zhang, H., Xue, S., Lwanga, E.H., Zomer, P., Ritsema, C.J. 605 and Geissen, V. (2018) Influence of microplastic addition on glyphosate decay and soil microbial 606 activities in Chinese loess soil. Environmental Pollution 242, 338-347. 
Figure 1

Glass tube set up drawing.

LDPE : Low Density Polyethylene mulch ; PAC : Pro-oxidant Additive Containing mulch ; Bio : Biodegradable mulch. Pesticides content was analysed in the solution $(A)$ and in the plastic (B). All experiments were performed in duplicate. Glass tubes were incubated at $35^{\circ} \mathrm{C}$ for 15 days.

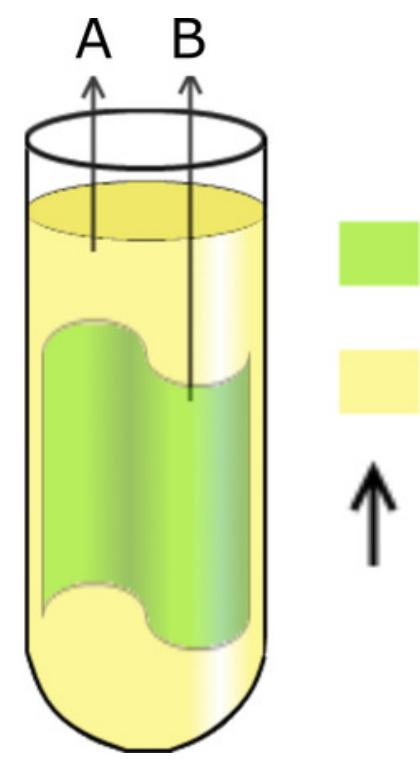

$3 \times 3 \mathrm{~cm}^{2}$ plastic piece: either LDPE, PAC or Bio $5 \mathrm{~mL}$ incubation solution ( $10 \%$ acetonitrile $+90 \% \mathrm{H}_{2} \mathrm{O}$ ): either with or without $1000 \mathrm{ng} \cdot \mathrm{mL}^{-1}$ of each active substance Pesticides extraction and analysis in the solution (A) or plastic (B) 


\section{Figure 2}

Sorption [\%] on each type of plastic: LDPE (blue), PAC (orange) and Biodegradable (red).

The box plot (horizontal lines) represents sorption for at least $25 \%, 50 \%$ and $75 \%$ of the active substances. The vertical black line ends represent the minimum and maximum values.

The cross is the mean sorption for all active substances. Different letters indicate significant differences among plastic types after a Kruskal-Wallis comparison at $p<0.05$.

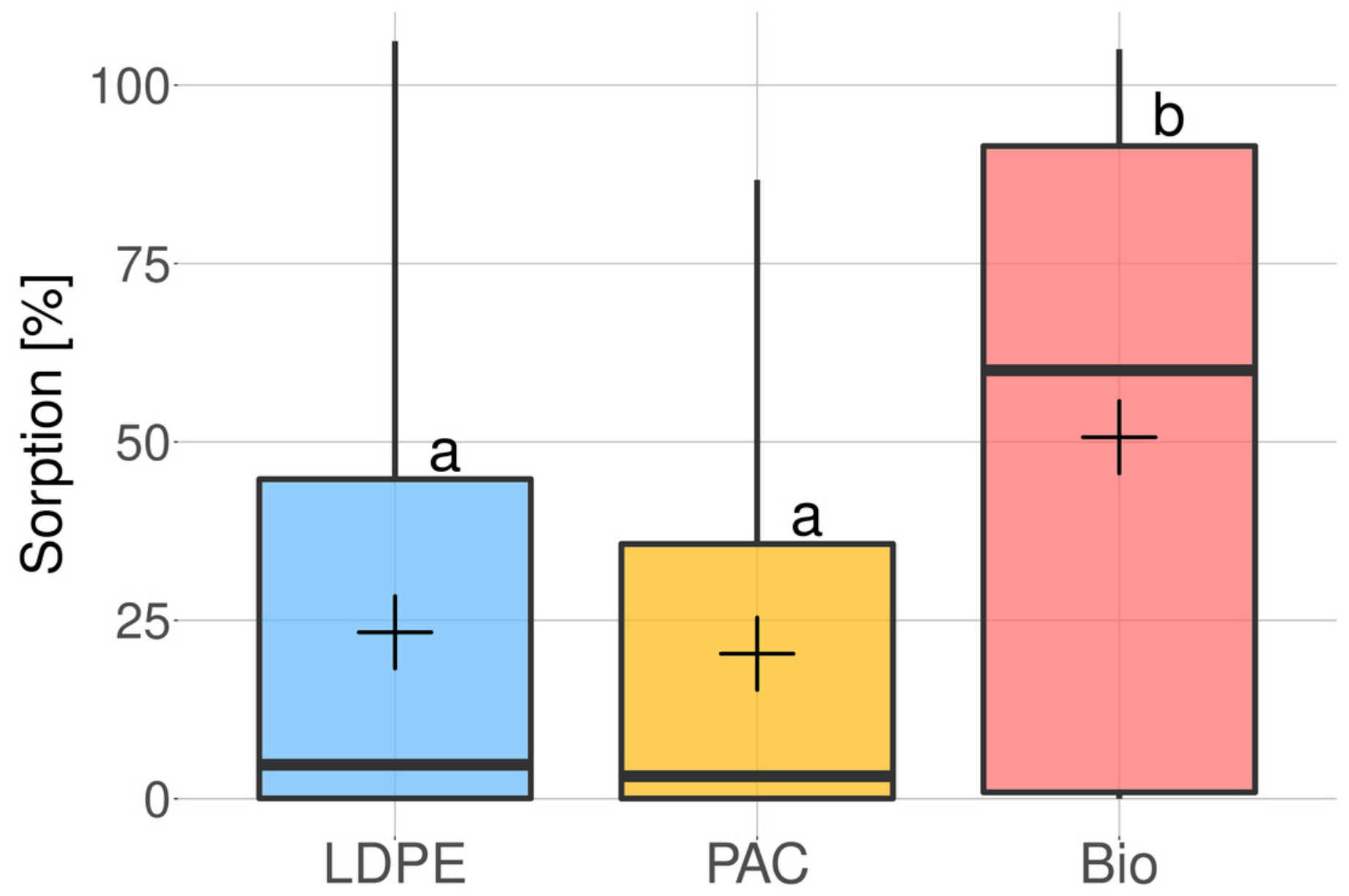




\section{Figure 3}

Mean sorption rate [\%] for each active substance on LDPE (blue), PAC (orange) and Biodegradable (red) plastic mulch.

Vertical black lines represent the measurement ranges ( $\min$ and max). Active substances are ordered according to increasing $\log \mathrm{P}$ (octanol-water partition coefficient).

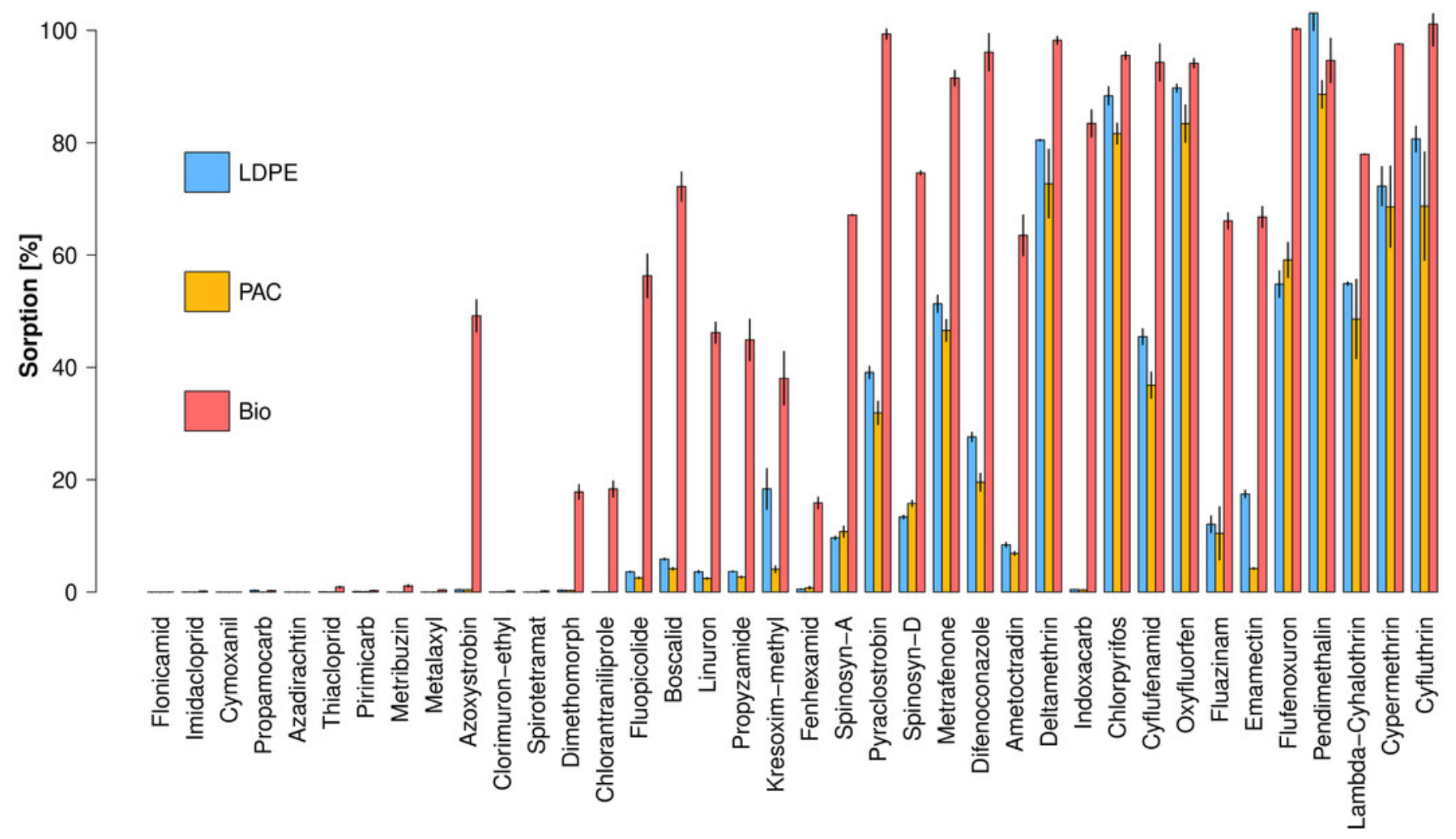


Figure 4

Sorption [\%] of active substances on LDPE, PAC and Bio mulch as a function of the active substances $\log P$ (octanol-water partition coefficient).

The black line is a regression calculated for sorption $>0: y=35 x-104 ; R^{2}=0.93 ; p<0.001$. 


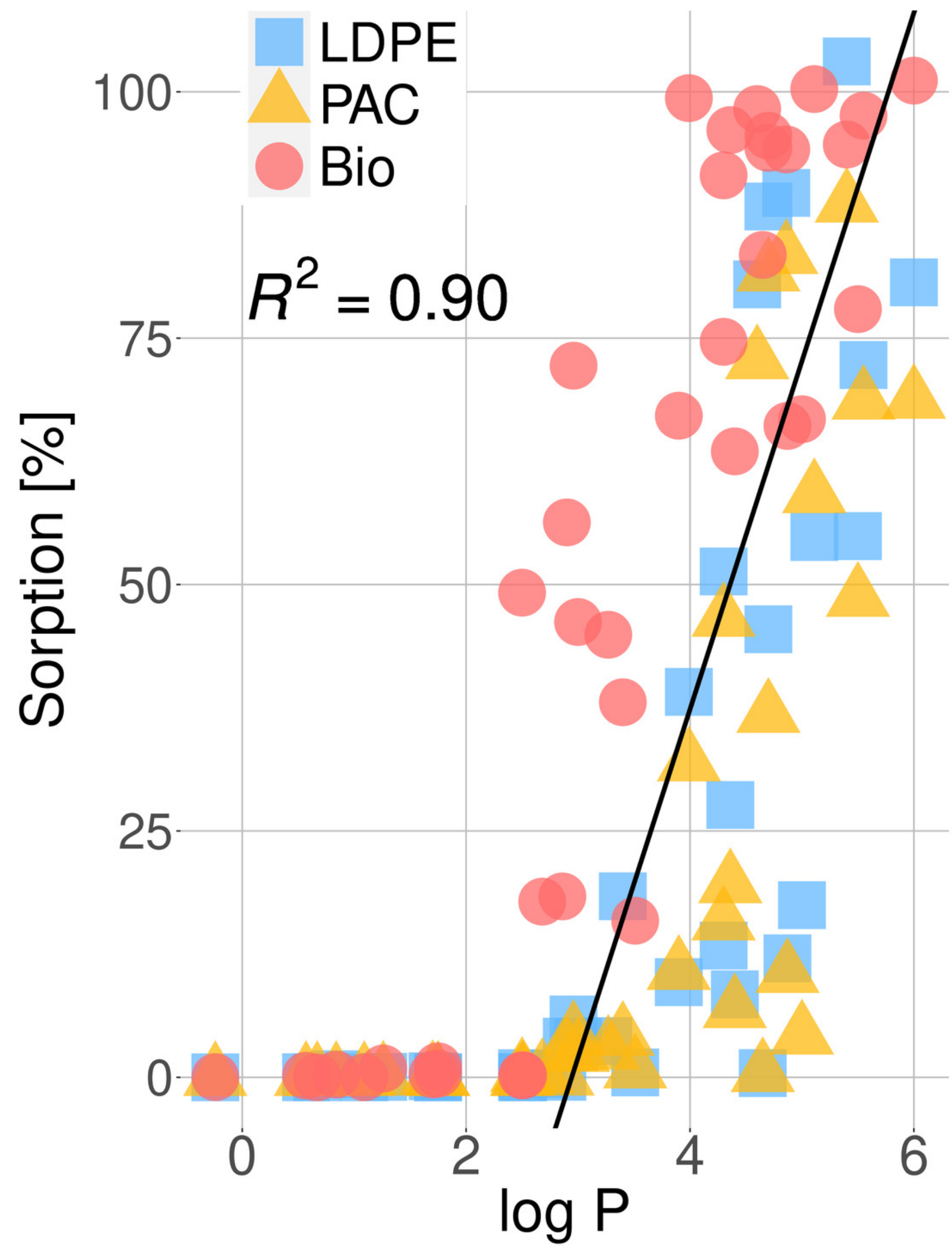


Figure 5

Decay reduction [\%] for active substances sorbed on plastics related to the sorption [\%] of active substances for the three types of plastic, LDPE (blue square), PAC (orange triangle) and Biodegradable (red circle).

The decay reduction [\%] is the difference between the decay of an active substance in presence of plastic and the decay of the same active substances without plastic. The black line is the regression calculated for sorption $>0: y=0.86 x-7 ; R^{2}=0.90 ; p<0.001$.

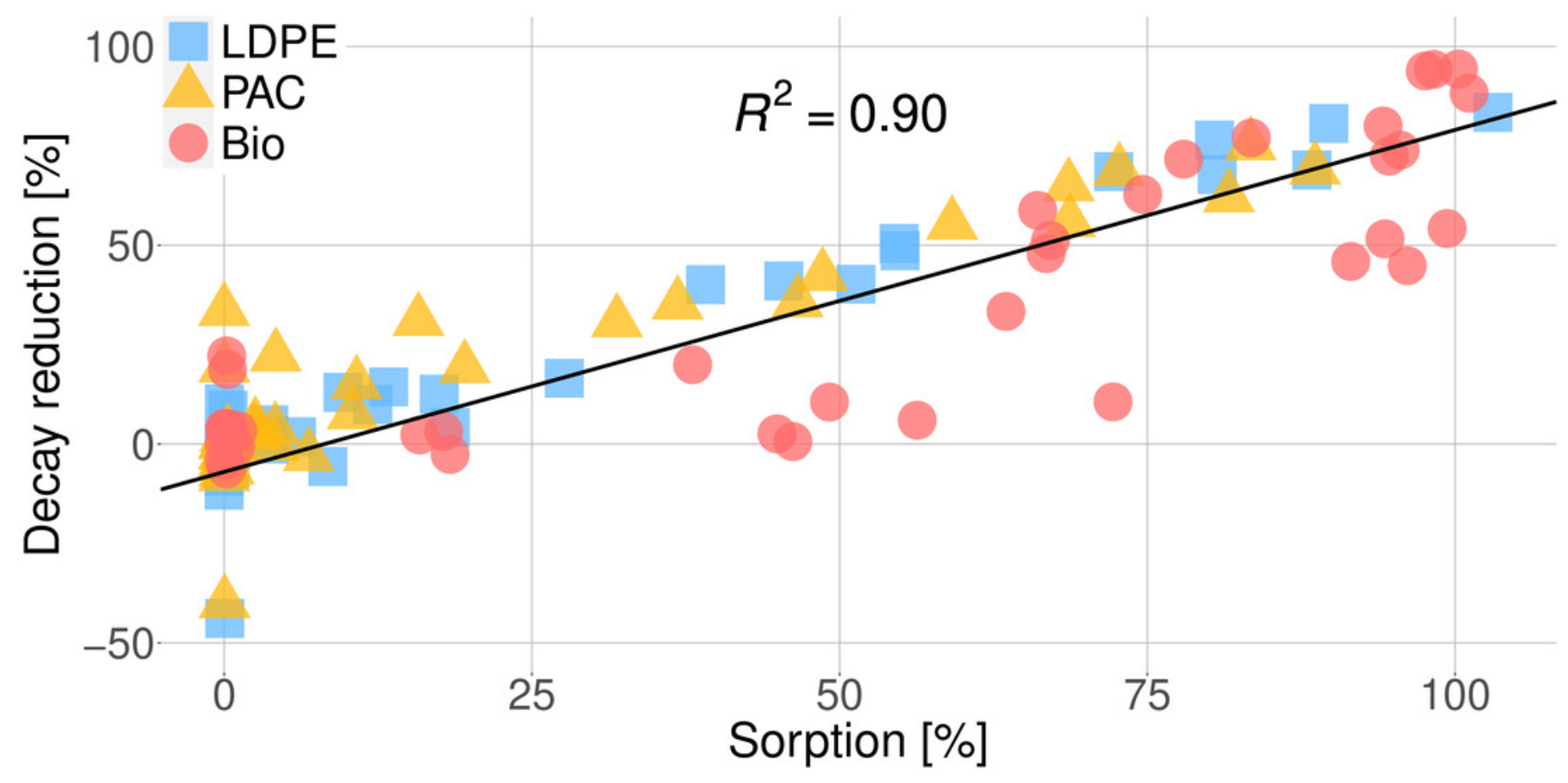




\section{Table $\mathbf{1}$ (on next page)}

Type of plastic mulch and incubation solution for the seven treatments.

LDPE : Low Density Polyethylene mulch ; PAC : Pro-oxidant Additive Containing mulch ; Bio :

Biodegradable mulch ; P : mixture containing the active substances ; W : mixture without the active substances. 


\begin{tabular}{|c|c|c|}
\hline Treatment & $\begin{array}{c}\text { Plastic } \\
\text { mulch }\end{array}$ & $\begin{array}{c}\text { Incubation solution } \\
\left(10 \% \text { acetonitrile and } 90 \% \mathrm{H}_{2} \mathrm{O}\right)\end{array}$ \\
\hline LDPE+P & LDPE & Active substances mixture \\
\hline PAC+P & PAC & Active substances mixture \\
\hline Bio+P & Bio & Active substances mixture \\
\hline LDPE+W & LDPE & No-active substances \\
\hline PAC+W & PAC & No-active substances \\
\hline Bio+W & Bio & No-active substances \\
\hline P & - & Active substances mixture \\
\hline
\end{tabular}

1 